\title{
Rare Presentation of a Metastatic Pancreatic Neuroendocrine Neoplasm Presenting with Atrial Flutter
}

\author{
Paraskevi C Fragkou ${ }^{1}$, Ioannis A Papadopoulos ${ }^{2}$, Antonios Papadopoulos ${ }^{1}$, Evangelos-Achilleas Kontoveros ${ }^{3}$, \\ Gregory Kaltsas ${ }^{4}$, Foula Vassilara ${ }^{2}$ \\ ${ }^{1}$ 4th Department of Internal Medicine, Attikon University Hospital, National and Kapodistrian University of Athens, Athens, Greece \\ ${ }^{2} 5$ th Department of Internal Medicine, Hygeia General Hospital, Athens, Greece \\ ${ }^{3}$ Ultrasound Department, Hygeia General Hospital, Athens, Greece \\ ${ }^{4}$ 1st Propaedeutic Department of Internal Medicine, Laikon General Hospital, National and Kapodistrian University of Athens, Athens, Greece
}

\section{Doi: 10.12890/2021_002216 - European Journal of Case Reports in Internal Medicine - @ EFIM 2021}

Received: $12 / 12 / 2020$

Accepted: $23 / 12 / 2020$

Published: 20/01/2021

How to cite this article: Fragkou P, Papadopoulos IA, Papadopoulos A, Kontoveros EA, Kaltsas G, Vassilara F. Rare presentation of a metastatic pancreatic neuroendocrine neoplasm presenting with atrial flutter. EJCRIM 2021;8: doi:10.12890/2020_002216.

Conflicts of Interests: The Authors declare that there are no competing interests.

This article is licensed under a Commons Attribution Non-Commercial 4.0 License

\section{ABSTRACT}

Pancreatic neuroendocrine neoplasms (PanNENs) rarely secrete serotonin, which is the main cause of carcinoid syndrome. One of its unusual manifestations is carcinoid heart disease or Hedinger's syndrome which is seldom accompanied by cardiac arrhythmias. We report the case of an 88-year-old woman who presented with recently experienced episodes of palpitations and a newly developed atrial flutter with a ventricular rate of 130 beats per minute. Echocardiography revealed thickened and tethered tricuspid and pulmonary valve leaflets causing severe valvular regurgitation and right ventricular dilatation. Episodes of intermittent diarrhoea over the previous 2 years were mentioned, making carcinoid syndrome our working diagnosis. The 5-hydroxyindoleacetic acid (5-HIAA) levels in a 24-hour urine collection specimen were elevated. Conventional imaging studies and a Ga-68 dodecane tetraacetic acid tyrosine-3-octreotate (DOTATATE) positron emission tomography/computer tomography (PET/CT) scan revealed the presence of a metastatic PanNEN arising from the pancreatic tail. The patient was managed with lanreotide and telotristat with remarkable improvement of her symptoms. To our knowledge, this is the first reported case of carcinoid syndrome presenting with atrial flutter as the initial symptom.

\section{LEARNING POINTS}

- Ultrasonography findings can indicate or lead to the diagnosis of carcinoid heart disease or Hedinger's syndrome.

- Clinicians should investigate rarer causes of atrial flutter when common ones are excluded.

- Even in advanced metastatic disease, complete remission of symptoms may be achieved with somatostatin analogues along with telotristat ethyl.

\section{KEYWORDS}

Neuroendocrine neoplasms, carcinoid heart disease, carcinoid syndrome, supraventricular tachycardia, atrial flutter

\section{INTRODUCTION}

The gastroenteropancreatic (GEP)-NENs are separated into two major categories: (a) well-differentiated neuroendocrine tumours (NETs), traditionally referred to as carcinoid tumours, and (b) poorly differentiated neuroendocrine carcinomas (NECs), which resemble small cell lung carcinoma. NENs are also classified by their secretory behaviour as 'functioning' or 'non-functioning' [1] 
Although well-differentiated NETs are found incidentally in pathology specimens, their clinical features, when present, are associated with the secretion of bioactive peptides and/or with the tumour bulk itself. Symptoms like flushing, diarrhoea and less commonly bronchospasm, are part of carcinoid syndrome, caused by hepatic and/or lung metabolic bypass of circulating peptides ${ }^{[2]}$. Carcinoid heart disease (CHD) may occur in patients with carcinoid syndrome. CHD is characterized by the formation of fibrous-like plaques at the endocardium causing fixation and thickening of the valvular leaflets mostly at the right-sided valves, leading to right heart failure. Ventricular tachycardia and atrial fibrillation have also been sporadically reported ${ }^{[3]}$. However, other arrhythmias and particularly atrial flutter have never been reported in the context of CHD. To our knowledge, we present the first reported case of an elderly woman presenting with atrial flutter as the initial symptom of carcinoid syndrome and CHD.

\section{CASE DESCRIPTION}

An 88-year-old woman presented with disturbing episodes of palpitations and mild dyspnoea during the last 48 hours. The patient was haemodynamically stable with a blood pressure of $178 / 98 \mathrm{mmHg}$ and a rhythmic pulse rate of 130 beats per minute. ECG demonstrated supraventricular tachycardia (SVT), consistent with atrial flutter. Continuous 48-hour ECG monitoring recorded five similar episodes of SVT per day. Transthoracic (TTE) (Fig. 1A,B) and transesophageal (TEE) echocardiograms revealed thickened and tethered tricuspid and pulmonary valve leaflets leading to severe valve regurgitation. Echocardiography also showed a dilated right ventricle with preserved systolic function and a left ejection fraction of $>55 \%$.

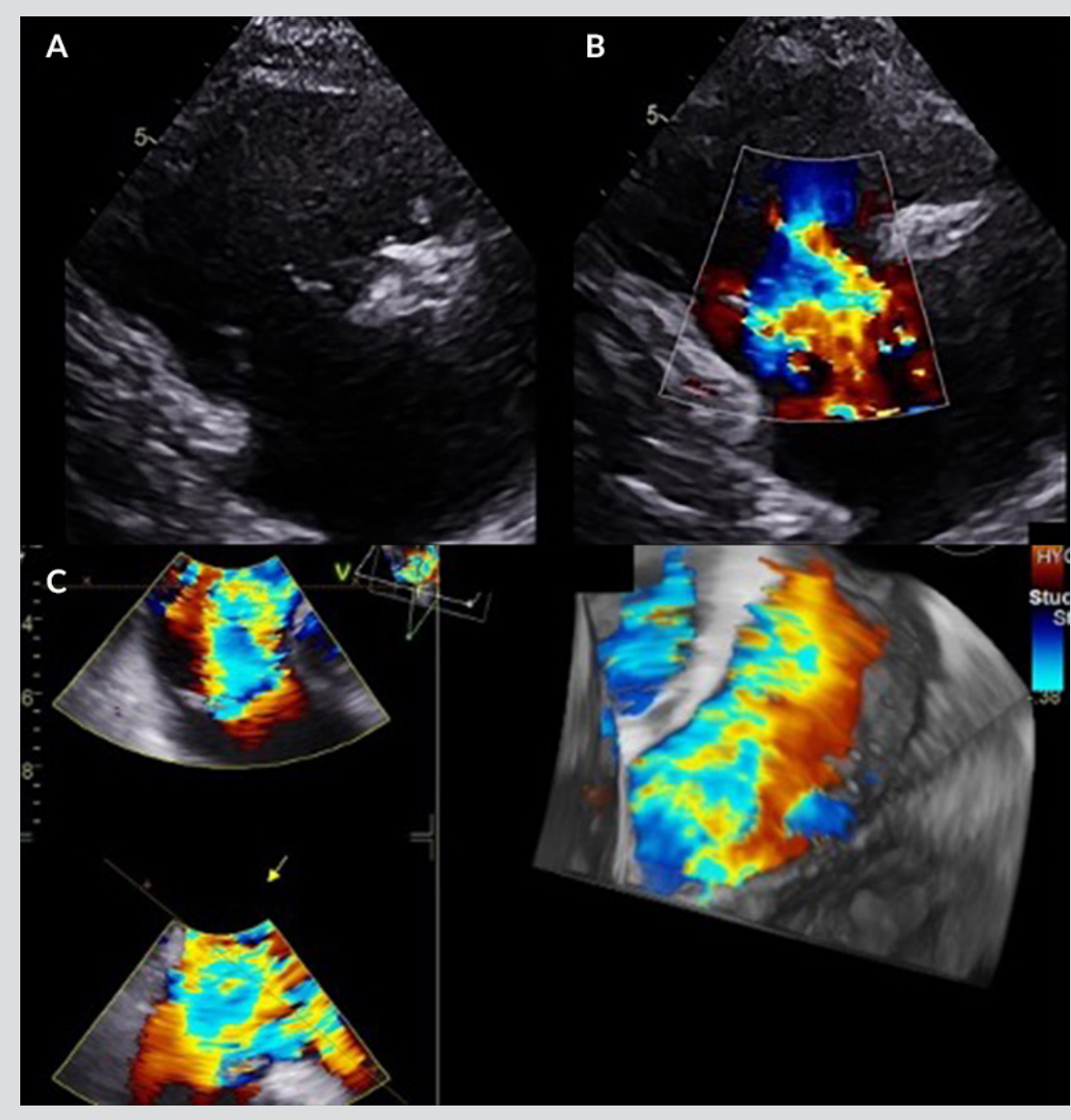

Figure 1. (A, B) Right ventricle inflow view at end systole in a transthoracic echocardiogram. Tricuspid valve leaflets appeared shortened, retracted and immobile leading to $(A)$ valve malcoaptation and $(B)$ subsequent severe regurgitation. (C) 3D full-volume colour acquisition of the transesophageal echocardiogram showing severe tricuspid regurgitation

Because of the above echocardiographic findings, the patient's history was taken in more detail. She said she had experienced intermittent episodes of diarrhoea over the last 2 years. A colonoscopy 2 years previously was reported as unremarkable.

5-Hydroxyindoleacetic acid (5-HIAA) and chromogranin A (CgA) levels in a 24-hour urine collection specimen were $69.1 \mathrm{mg} / 24 \mathrm{~h}$ (reference range $<15 \mathrm{mg} / 24 \mathrm{~h}$ ) and $302 \mathrm{ng} / \mathrm{ml}$ (reference range $<120 \mathrm{ng} / \mathrm{ml}$ ), respectively, confirming our working diagnosis of carcinoid syndrome. 
Imaging with CT and MRI revealed a pancreatic neoplasm with liver metastases. A Ga-68DOTATATE PET/CT scan established the presence of multiple foci characterized by overexpression of somatostatin receptors at thoracic cage bones, vertebrae, pelvic bones, liver and mesentery (Fig. 2). The scan also revealed a solid mass at the pancreatic tail that was thought to be the primary site of the metastatic carcinoid tumour. As the patient refused biopsy, she was managed empirically with lanreotide $120 \mathrm{mg}$ monthly and telotristat ethyl $250 \mathrm{mg}$ three times daily, with impressive response to the treatment and a stable condition during 16 months of follow-up.

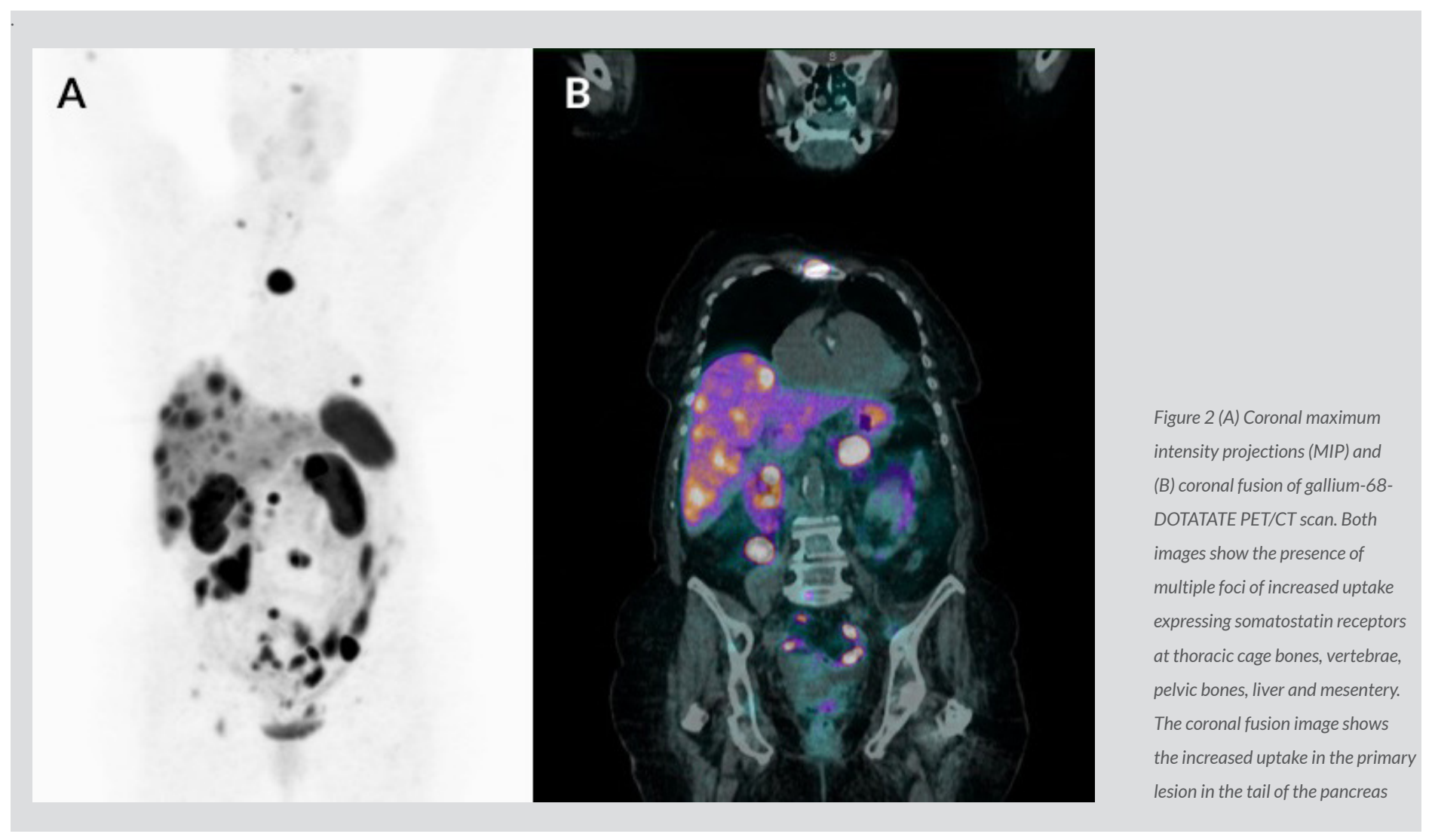

\section{DISCUSSION}

We present the first reported case of a metastatic PanNEN causing carcinoid syndrome that presented originally as atrial flutter. PanNENs account for $30 \%$ of all GEP-NENs. Most of them (70-90\%) are non-functioning and are discovered incidentally. When PanNENs are functioning they mainly secrete insulin, glucagon and gastrin. More rarely, as in our case, they secrete serotonin or 5-hydroxytryptamine $(5-\mathrm{HT})$, which is the main cause of carcinoid syndrome ${ }^{[4]}$. In most patients, there is increased urinary excretion of 5-HIAA, a degradation product of serotonin.

The non-specific symptoms early in the course of NENs and, most importantly, the low index of suspicion contribute to delayed diagnosis. Consequently, most cases are metastatic at the time of diagnosis. Well-differentiated NETs are mostly found incidentally in pathology specimens. When they produce symptoms, abdominal pain and specific manifestations according to secretion of bioactive substances are their main characteristics. They can secrete insulin, gastrin, glucagon, serotonin (5-HT), 5-hydroxytryptophan (5-HTP), prostaglandins, bradykinin, histamine, somatostatin, growth hormone (GH), adrenocorticotropin (ACTH), calcitonin, CgA and peptide YY ${ }^{[4]}$. Carcinoid syndrome (CS) is the most common secretory paraneoplastic syndrome. Carcinoid syndrome is characterized by diarrhoea, flushing, abdominal pain, bronchospasm and CHD.

In our patient, the main feature suggesting the diagnosis was the characteristic echocardiography finding of Hedinger's syndrome, which presents in up to $20 \%$ of patients with carcinoid syndrome ${ }^{[5]}$. $\mathrm{CHD}$ is characterized by the development of fibrous-like plaques on the valves, endocardium and sometimes on the vena cava endothelium, leading to thickening and fixation of the leaflets. This, in turn, causes insufficiency of the affected valvular and subvalvular apparatus. The bioactive substances secreted by the tumour promote the activation and proliferation of fibroblasts and smooth muscle cells by the overproduction of transforming growth factor- $\beta 1$ (TGF- $\beta 1$ ) and the expression of inflammatory cytokines through activation of $5-\mathrm{HT} 2 \mathrm{~B}$ receptors which are abundant in cardiac valves ${ }^{[5,6]}$. This condition 
primarily affects the right-sided chambers and valves because high levels of the biomolecules are not degraded by the liver and lungs. Other conditions associated with CHD that have been sporadically reported are restrictive cardiomyopathy and constrictive pericarditis. Cardiac arrhythmias have been described mostly during the carcinoid crisis, a life-threatening condition where massive amounts of serotonin and other vasoactive molecules are suddenly released during stress, chemotherapy or surgery; these peptides precipitate bronchospasm, tachycardia, flushing and hypotension. Outside the context of carcinoid crisis, only a few cases have been reported with carcinoid-mediated dysrhythmias like ventricular tachycardia or atrial fibrillation due to the proarrhythmic effects of serotonin ${ }^{[3]}$. Atrial flutter has not been reported as the presenting manifestation of CHD. As atrial flutter is frequently attributed to heart failure, diabetes, hypertension, coronary artery disease, acute illness, alcoholism or thyroid dysfunction, it is doubtful whether we would have investigated the possibility of carcinoid syndrome without the suggestive echocardiography findings in this patient.

The diagnosis of carcinoid syndrome is based on biomarkers and imaging studies targeting specific receptors on carcinoid cells. 5-HIAA and $\mathrm{CgA}$ are the main biomarkers utilized in diagnosis and follow-up of the tumour.

Computed tomography and currently, diffusion-weighted imaging (DWI) with MRI (DW-MRI), as well as endoscopic ultrasound are used as essential modalities in the detection and localization of $\mathrm{NENs}^{[4]}$. Octreotide scanning should be considered as a useful modality when extremely sensitive PET/CT with Ga 68-DOTA-somatostatin analogue is not available. Treatment options for carcinoid syndrome include the somatostatin analogues octreotide and lanreotide, which are effective even in metastatic cases ${ }^{[4]}$. Our patient was managed with lanreotide along with telotristat ethyl, an inhibitor of the tryptophan hydroxylase with peripheral action, which is used as an adjuvant anti-diarrhoeal agent for carcinoid-associated diarrhoea ${ }^{[7]}$.

\section{REFERENCES}

1. WHO Classification of Tumours Editorial Board. Digestive System Tumours, WHO Classification of Tumours. 5th ed. Lyon, France: IARC Press; 2019.

2. Ito T, Lee L, Jensenc RT. Carcinoid-syndrome: recent advances, current status and controversies. Curr Opin Endocrinol Diabetes Obes 2018;25:22-35.

3. Langer C, Piper C, Vogt J, Heintze J, Butz T, Lindner O, et al. Atrial fibrillation in carcinoid heart disease: the role of serotonin. A review of the literature. Clin Res Cardiol 2007;96:114-118.

4. Pavel M, Oberg K, Falconi M, Krenning EP, Sundin A, Perren A, et al. Gastroenteropancreatic neuroendocrine neoplasms: ESMO Clinical Practice Guidelines for diagnosis, treatment and follow up. Ann Oncol 2020;31(7):844-860.

5. Davar J, Connolly HM, Caplin ME, Pavel M, Zacks J, Bhattacharyya S, et al. Diagnosing and managing carcinoid heart disease in patients with neuroendocrine tumors: an expert statement. J Am Coll Cardiol 2017;69:1288-1304.

6. Mota JM, Sousa LG, Riechelmann RP. Complications from carcinoid syndrome: review of the current evidence. Ecancermedicalscience 2016 Aug 8;10.662. https://doi. org/10.3332/ecancer.2016.662.

7. Tsoli M, Chatzellis E, Koumarianou A, Kolomodi D, Kaltsas G. Current best practice in the management of neuroendocrine tumors. Ther Adv Endocrinol Metab 2019;10:1-18. 